\title{
Hybrid Predictive Control Based on High-Order Differential State Observers and Lyapunov Functions for Switched Nonlinear Systems
}

\author{
Baili Su${ }^{1}$, Guoyuan $\mathbf{Q i}^{2}$, Barend J. van $\mathrm{Wyk}^{2}$ \\ ${ }^{1}$ School of Electrical Information and Automation, Qufu Normal University, Qufu, China \\ ${ }^{2}$ F'SATIE and Department of Electrical Engineering, Tshwane University of Technology, Pretoria, South Africa \\ Email: guoyuanqi@gmail.com, vanwykb@gmail.com
}

Received April 26, 2013; revised May 26, 2013; accepted June 4, 2013

Copyright (C) 2013 Baili Su et al. This is an open access article distributed under the Creative Commons Attribution License, which permits unrestricted use, distribution, and reproduction in any medium, provided the original work is properly cited.

\begin{abstract}
In this paper, a hybrid predictive controller is proposed for a class of uncertain switched nonlinear systems based on high-order differential state observers and Lyapunov functions. The main idea is to design an output feedback bounded controller and a predictive controller for each subsystem using high-order differential state observers and Lyapunov functions, to derive a suitable switched law to stabilize the closed-loop subsystem, and to provide an explicitly characterized set of initial conditions. For the whole switched system, based on the high-order differentiator, a suitable switched law is designed to ensure the whole closed-loop's stability. The simulation results for a chemical process show the validity of the controller proposed in this paper.
\end{abstract}

Keywords: Switched System; Lyapunov Function; High Order Differentiator; Control Constraint; Output Feedback; Model Predictive Control; Stable Region

\section{Introduction}

Switched system is a typical hybrid dynamic system made up of some subsystems and a switched law. In recent years, the stabilization of constrained switched systems became an attractive research subject [1].

Model predictive control (MPC) is a receding horizon control (RHC) method to handle constraints within an optimal control setting [2]. There have been many results to show the performance of constrained MPC [3]. In MPC design, the initial feasibility of the optimization problem is always assumed. Due to uncertainties and constraints of the practical process, this assumption may not be satisfied. Furthermore, the set of initial conditions, starting from where a given MPC formulation is guaranteed to be feasible, has not been explicitly characterized.

In recent years, controller design methods based on Lyapunov functions have been developed, which can give an explicitly characterized set of initial conditions from which the closed-loop system is stable [4]. By embedding the Lyapunov-based design methods into the MPC design, we can obtain the set of initial conditions from where the closed-loop system is stable. In refs. [5,6], two Lyapunov-based predictive controllers were derived for constrained nonlinear systems. In refs. [7,8], two Lyapunov-based predictive controllers were proposed for constrained switched systems and constrained switched systems with uncertainties, respectively. In these papers, the states of the system are observable.

However, in real processes the system's states are often not measurable, and hence, state-feedback controllers and switched laws cannot be realized. One of the methods to overcome this difficulty is to construct a state observer to estimate the states for constructing the controller and switched law. In ref. [9], an output feedback bounded controller was given for a class of nonlinear systems which was not switched system. In ref. [10], for a kind of nonlinear switched systems without uncertainties and disturbance, a bounded nonlinear controller was given. But it was not guaranteed to be optimal with respect to an arbitrary performance criterion which incurporates requested performance in the design. In ref. [11], a hybrid output feedback predictive controller was proposed for a class of switched nonlinear systems without uncertainties. In papers [9-11], the processes' states were estimated using a high-gain observer, but many adjustable parameters of the observer need to be chosen expe- 
rientially. Sometimes the wrong selection of parameters can cause stability problems and an undesired transient performance of the observer. In refs. [12-14], a high-order differential state observer was designed to estimate the states of a nonlinear system. Theoretically the parameters are chosen according to the performance and stability of the observer and theoretically few parameters with explicit meanings have to be selected based on the performance and stability of the observer.

In this paper, an output feedback hybrid predictive controller is proposed for a class of uncertain switched nonlinear systems based on high-order differential state observers and Lyapunov functions. The main idea is to design a hybrid predictive controller based on Lyapunov functions and high-order differential state observers, which switches between a bounded feedback controller and a predictive controller for each subsystem, and to provide an explicitly characterized set of initial conditions to stabilize the closed-loop subsystem. Here, we use high-order differentiators as state observers. This high-order differential state observer has simple structure with few parameters. A suitable switched law based on the high-order differentiator is designed to guarantee the whole closed-loop system's stability. Finally, the simulation results for a chemical process show the validity of the procedure proposed in this paper.

\section{Problem Description}

Consider the constrained switched nonlinear system

$$
\begin{aligned}
x(t)= & f_{\sigma(t)}(x(t))+G_{\sigma(t)}(x(t)) u_{\sigma(t)}(t) \\
& +W_{\sigma(t)}(x(t)) \boldsymbol{\theta}_{\sigma(t)}(t) \\
y(t)= & h_{\sigma(t)}(x) \\
x(t) \in & X \subseteq R^{n}, u_{\sigma}(t) \in U_{\sigma} \subseteq R^{m}, \theta_{\sigma} \in \Theta_{\sigma} \subseteq R
\end{aligned}
$$

where $\boldsymbol{x}(t)=\left[x_{1}, \cdots, x_{n}\right]^{\mathrm{T}} \in X \subseteq R^{n}$ denotes the vector of continuous-time state variables, $X$ is the set containing the states;

$\boldsymbol{u}_{\sigma}(t)=\left[u_{\sigma}^{1}(t), \cdots, u_{\sigma}^{m}(t)\right]^{\mathrm{T}} \in U_{\sigma} \subset R^{m}$ denotes the vector of manipulated inputs taking values in a nonempty compact subset $U_{\sigma}=\left\{\boldsymbol{u}_{\sigma} \in R^{m}:\left\|\boldsymbol{u}_{\sigma}\right\| \leq u_{\sigma}^{\max }\right\}$, where $\|$. is the Euclidian norm, and $u_{\sigma}^{\max }>0$ is the magnitude of the constraints. $y \in R$ denotes the measured output; $h_{\sigma(t)}(\boldsymbol{x}) \in R$ is a sufficiently smooth function;

$\boldsymbol{\theta}_{\sigma}(t)=\left[\theta_{\sigma}^{1}(t), \cdots, \theta_{\sigma}^{q}(t)\right] \in \Theta_{\sigma} \subseteq R^{q}$ denotes the bounded uncertain parameter vector taking values in a nonempty compact subset $\Theta_{\sigma}=\left\{\boldsymbol{\theta}_{\sigma} \in R^{q}:\left\|\boldsymbol{\theta}_{\sigma}\right\| \leq \theta_{\sigma}^{b}\right\}$; $\sigma:[0, \infty) \rightarrow K$ is the switching signal assuming to be a piece-wise continuous (from the right) function of time, i.e., $\sigma\left(t_{k}\right)=\lim _{t \rightarrow t_{k}^{+}} \sigma(t)$ for all $k$, implying that only a finite number of switches is allowed on any finite inter- val of time, and $p$ is the number of modes of the switched system. Throughout this paper, we use the notations $t_{k_{r}^{\text {in }}}$ and $t_{k_{r}^{\text {out }}}$ to denote the time at which, for the rth time, the $k$ th $^{k_{r}}$ subsystem is switched in and out, respectively, i.e., $\sigma\left(t_{k_{r}^{+}}^{+}\right)=\sigma\left(t_{k_{r}^{\text {out }}}^{-}\right)=k$. With this notation, it is understandable that the continuous state evolves according to $\dot{\boldsymbol{x}}=\boldsymbol{f}_{k}(\boldsymbol{x})+\boldsymbol{G}_{k}(\boldsymbol{x}) \boldsymbol{u}_{k}+\boldsymbol{W}_{k}(\boldsymbol{x}) \boldsymbol{\theta}_{k}$, $y=h_{k}(\boldsymbol{x})$, for $t_{k_{r}^{\text {in }}} \leq t \leq t_{k_{r}^{\text {out }}} \cdot T_{k_{r}^{\text {in }}}=\left\{t_{k_{1}^{\text {in }}}, t_{k_{2}^{\text {in }}}, \cdots\right\}$ and $T_{k_{r}^{\text {out }}}=\left\{t_{k_{1}^{\text {out }}}, t_{k_{2}^{\text {out }}}, \cdots\right\}$ denote the set of switching times at which the $k$ th subsystem is switched in and out, respectively. It is assumed that all entries of the vector functions $\boldsymbol{f}_{k}(\boldsymbol{x})$, the $n \times m$ matrices $\boldsymbol{G}_{k}(\boldsymbol{x})$ and the $n \times q$ matrices $\boldsymbol{W}_{k}(\boldsymbol{x})$ are sufficiently smooth and that $\boldsymbol{f}_{k}(\mathbf{0})=\mathbf{0}$ for all $k \in K$. In this paper, the notation $L_{f} \bar{h}$ denotes the standard Lie derivative of a scalar function $\bar{h}(\boldsymbol{x})$ with respect to the vector function

$$
\begin{aligned}
& \boldsymbol{f}(\boldsymbol{x}): L_{\boldsymbol{f}} \bar{h}(\boldsymbol{x})=\left(\frac{\partial \bar{h}}{\partial \boldsymbol{x}}\right) \boldsymbol{f}(\boldsymbol{x}) \text {, and } \\
& \limsup _{t \rightarrow \infty} \boldsymbol{f}(\boldsymbol{x}(t))=\lim _{t \rightarrow \infty}\left\{\sup _{\tau \geq t} \boldsymbol{f}(\boldsymbol{x}(\tau))\right\} .
\end{aligned}
$$

The objective of this paper is to design a nonlinear output feedback predictive controller based on Lyapunov functions and a high order differential state observer for the case where state measurements are not available for each mode of the uncertain switched nonlinear system given by Equation (1). Then, for the whole switched system, based on state estimations, a suitable switched law is designed to ensure the whole closed-loop system's stability.

\section{Preliminaries}

\subsection{High-Order Differential State Observers}

In order to construct an output feedback controller to stabilize the controlled system (1), we use high-order differential state observers [12-14] to estimate the unmeasurable states of the system (1).

Firstly, we give some assumptions.

Assumption 1: Consider system (1), for every $k \in K$, there exist an integer $r_{k}$ and a set of invertible coordinates

$$
\left[\begin{array}{c}
\boldsymbol{\xi} \\
\boldsymbol{\eta}
\end{array}\right]=\left[\begin{array}{c}
\xi_{1} \\
\vdots \\
\xi_{r_{k}} \\
\eta_{1} \\
\vdots \\
\eta_{n-r_{k}}
\end{array}\right]=\chi_{k}(\boldsymbol{x})=\left[\begin{array}{c}
h_{k}(\boldsymbol{x}) \\
\vdots \\
L_{\boldsymbol{f}_{k}}^{r_{k}-1} h_{k}(\boldsymbol{x}) \\
\chi_{k, 1}(\boldsymbol{x}) \\
\vdots \\
\chi_{k, n-r_{k}}(\boldsymbol{x})
\end{array}\right]
$$


where $\chi_{k, 1}(\boldsymbol{x}), \cdots, \chi_{k, n-r_{k}}(\boldsymbol{x})$ are nonlinear scalar functions of $x$, such that the system (1) takes the form

$$
\begin{aligned}
& \dot{\xi}_{1}=\xi_{2} \\
& \quad \vdots \\
& \dot{\xi}_{r_{k}-1}=\xi_{r_{k}} \\
& \dot{\xi}_{r_{k}}=L_{f_{k}}^{r_{k}} h_{k}\left(\chi^{-1}(\xi, \boldsymbol{\eta})\right)+L_{\boldsymbol{G}_{k}} L_{f_{k}}^{r_{k}-1} h_{k}\left(\chi^{-1}(\xi, \boldsymbol{\eta})\right) \boldsymbol{u}_{k} \\
& \quad+L_{\boldsymbol{W}_{k}} L_{\boldsymbol{f}_{k}}^{r_{k}-1} h_{k}\left(\chi^{-1}(\xi, \boldsymbol{\eta})\right) \boldsymbol{\theta}_{k} \\
& \dot{\eta}_{1}=\Psi_{k, 1}\left(\xi, \boldsymbol{\eta}, \boldsymbol{\theta}_{k}\right) \\
& \quad \vdots \\
& \dot{\eta}_{n-r_{k}}=\Psi_{k, n-r_{k}}\left(\boldsymbol{\xi}, \boldsymbol{\eta}, \boldsymbol{\theta}_{k}\right) \\
& y=\xi_{1}
\end{aligned}
$$

where $\boldsymbol{x}=\chi^{-1}\left(\boldsymbol{\xi}_{k}, \boldsymbol{\eta}_{k}, \boldsymbol{\theta}_{k}\right)$, $L_{\boldsymbol{G}_{k}} L_{f_{k}}^{r_{k}-1} h_{k}\left(\chi^{-1}\left(\boldsymbol{\xi}, \boldsymbol{\eta}, \boldsymbol{\theta}_{k}\right)\right) \neq 0, \forall \boldsymbol{x} \in R^{n}$, and

$\Psi_{k, 1}, \cdots, \Psi_{k, n-r_{k}}$ are nonlinear functions describing the corresponding evolution of the kth inverse dynamics mode, and $\boldsymbol{\xi}_{k} \rightarrow 0, \quad \boldsymbol{\eta}_{k} \rightarrow 0$ if and only if $\boldsymbol{x} \rightarrow 0$.

Assumption 2: The dynamical $\boldsymbol{\eta}$-subsystem in (3)

$$
\dot{\eta}=\Psi_{k}\left(e, \boldsymbol{\eta}, \boldsymbol{\theta}_{k}, \bar{v}\right)
$$

is input-to-state stable (ISS) [9], where $\boldsymbol{\eta}=\left[\eta_{1}, \cdots, \eta_{n-r_{k}}\right]^{\mathrm{T}}$ and $\Psi_{k}=\left[\Psi_{k, 1}, \cdots, \Psi_{k, n-r_{k}}\right]^{\mathrm{T}}$.

The following assumptions are given to reduce the influence of uncertainties.

Assumption 3: There exists a known constant $\theta_{b k}$ such that $\left\|\theta_{k}\right\|=\theta_{b k}$.

Assumption 4: For each $k \in K$, a control Lyapunov function $V_{k}$ exists.

Before designing the output feedback controller, we have to revise Assumption 1.

Assumption 5: There exists an invertible coordinate transformation $x=\chi^{-1}\left(\xi_{k}, \eta_{k}\right)$, such that system (1) takes the form similar to (3) in which the $\eta_{k}$-subsystem take the following form

$$
\dot{\eta}=\Psi_{k}(\xi, \boldsymbol{\eta})
$$

This formula is different from formula (4) since it does not depend on the uncertain parameter $\theta_{k}$. We also assume this subsystem is ISS stable.

In order to construct a controller to stabilize the controlled system (1), we use high-order differential state observers [12-14] to estimate the un-measurable states of system (1). The high-order differential state observer for each mode can be described as

$$
\sum:\left\{\begin{array}{l}
\dot{\tilde{y}}_{i}=\tilde{y}_{i+1}+a_{k, i}\left(y-\tilde{y}_{1}\right), \quad i=1, \cdots, r_{k}-1 \\
\dot{\tilde{y}}_{r_{k}}=a_{k, r_{k}}\left(y-\tilde{y}_{1}\right)
\end{array}\right.
$$

$$
\left\{\begin{array}{l}
\hat{y}_{1}=\tilde{y}_{1} \\
\hat{\boldsymbol{y}}_{i+1}=\tilde{y}_{i+1}+a_{k, i}\left(y-\tilde{y}_{1}\right), \quad i=1, \cdots, r_{k}-1
\end{array}\right.
$$

where $a_{k, i}=K_{k} C_{r_{k}-1}^{i-1} a_{k}^{i-1}, i=1, \cdots, r_{k}$

$$
K_{k}=\frac{\left(r_{k} a_{k}\right)^{r_{k}}}{\left(\left(r_{k}-1\right) a_{k}\right)^{r_{k}-1}}=\frac{r_{k}^{r_{k}} a_{k}}{\left(r_{k}-1\right)^{r_{k}-1}}
$$

Here $C_{j}^{i}$ denotes the combination expression and only one parameter $a_{k}$ is adjustable, all other parameters $a_{k, i}$ can be calculated using $r_{k}$ and $a_{k}$. Clearly, $y$ is the only external input of system $\sum$, so we can obtain $\tilde{y}_{1}, \tilde{y}_{2}, \cdots, \tilde{y}_{r_{\mathrm{k}}}$ based on the measured signal $y$ via (6), and further calculate the estimated derivatives $\hat{y}_{1}, \cdots, \hat{y}_{r_{k}}$ via (7). Note that the HOD is independent of the model of the original system (1).

Proposition 1. The HOD does not rely on the model of the estimated system, parameters are chosen using (8), and has following characteristics:

1) The HOD is an asymptotically stable system.

2) $\lim _{t \rightarrow \infty} \hat{y}_{i}=y^{(i-1)}=x_{i}, i=1, \cdots, r_{k}$

\subsection{State Feedback Bounded Controller Based on Lyapunov Functions}

We recall the design of a state feedback bounded controller to obtain the set of initial conditions from which the system is stable [9].

Define the tracking error variables $e_{i}=\xi_{i}-v^{(i-1)},\left(i=1, \cdots, r_{k}\right)$ and the tracking error vector $\boldsymbol{e}=\left[e_{1}, e_{2}, \cdots, e_{r_{k}}\right]^{\mathrm{T}}, \overline{\boldsymbol{v}}=\left[v, v^{(1)}, \cdots, v^{\left(r_{k}-1\right)}\right]^{\mathrm{T}}$ is the reference input vector, where $v$ is a reference input and $v^{(i)}$ is its ith time derivative. Then the $\xi_{k}$-subsystem in system (3) can be re-written as

$$
\begin{aligned}
& \dot{\boldsymbol{e}}_{k}=\overline{\boldsymbol{f}}_{k}\left(\boldsymbol{e}_{k}, \overline{\boldsymbol{v}}\right)+\overline{\boldsymbol{G}}_{k}\left(\boldsymbol{e}_{k}, \overline{\boldsymbol{v}}\right) \boldsymbol{u}_{k}+\overline{\boldsymbol{H}}_{k}\left(\boldsymbol{e}_{k}, \overline{\boldsymbol{v}}\right) \boldsymbol{\theta}_{k}, \\
& k=1, \cdots, p
\end{aligned}
$$

where $\overline{\boldsymbol{f}}_{k}(\boldsymbol{e}, \overline{\boldsymbol{v}})=\boldsymbol{A} \boldsymbol{e}+\boldsymbol{b} L_{f_{k}}^{r_{k}} h_{k}\left(\chi^{-1}(\boldsymbol{e}, \overline{\boldsymbol{v}})\right)$ is a $r_{k} \times 1$ vector function, $\overline{\boldsymbol{G}}_{k}(\boldsymbol{e}, \overline{\boldsymbol{v}})=\boldsymbol{b} L_{\boldsymbol{G}_{k}} L_{f_{k}}^{r_{k}-1} h_{k}\left(\chi^{-1}(\boldsymbol{e}, \overline{\boldsymbol{v}})\right)$ is a $r_{k} \times m$ matrix function, $\overline{\boldsymbol{H}}_{k}\left(\boldsymbol{e}_{k}, \overline{\boldsymbol{v}}\right)=\boldsymbol{b} L_{W_{k}} L_{f_{k}}^{r_{k}-1} h\left(\chi^{-1}\left(\boldsymbol{e}_{k}, \overline{\boldsymbol{v}}\right)\right)$ is a $r_{k} \times q$ matrix function, and

$$
\boldsymbol{A}=\left[\begin{array}{ccccc}
0 & 1 & 0 & \cdots & 0 \\
0 & 0 & 1 & \cdots & 0 \\
\vdots & \vdots & \vdots & \ddots & \vdots \\
0 & 0 & 0 & \cdots & 1 \\
0 & 0 & 0 & \cdots & 0
\end{array}\right]_{r_{k} \times r_{k}}, \quad \boldsymbol{b}=\left[\begin{array}{c}
0 \\
\vdots \\
0 \\
1
\end{array}\right]_{r_{k} \times 1} .
$$

The Lyapunov function is chosen as $\bar{V}_{k}=\boldsymbol{e}^{\mathrm{T}} \boldsymbol{P}_{k} \boldsymbol{e}$, where the positive-definite matrix $\boldsymbol{P}_{k}$ is chosen to sat- 
isfy the following Ricatti inequality

$$
\boldsymbol{A}^{\mathrm{T}} \boldsymbol{P}_{k}+\boldsymbol{P}_{k} \boldsymbol{A}-\boldsymbol{P}_{k} \boldsymbol{b} \boldsymbol{b}^{\mathrm{T}} \boldsymbol{P}_{k}<0
$$

Definition 1. $\alpha(\cdot): R^{+} \rightarrow R^{+}$is a class $K$ function if it is a strictly increasing function satisfying $\alpha(0)=0$.

Definition 2. Function $\beta: R^{+} \times R^{+} \rightarrow R^{+}$is a class $K L$ function, if $\forall t \geq 0, \beta(\cdot, t)$ is non-increasing, and $\lim _{s \rightarrow 0^{+}} \beta(s, t)=0 ; \forall s \geq 0, \beta(s, \cdot)$ is non-increasing, and $\lim _{t \rightarrow \infty} \beta(s, t)=0$.

Choose a class $K$ function $\bar{\alpha}_{k}(\cdot)$ that satisfies $\bar{\alpha}_{k}(\|\boldsymbol{x}\|) \leq V_{k}(\boldsymbol{x})$ and define the set

$\Omega_{k, b}\left(u_{k}^{\max }\right)=\left\{\boldsymbol{x} \in R^{n}: V_{k}(\boldsymbol{x}) \leq \delta_{k, b}\right\}$, where $\delta_{k, b}<\delta_{k, x}$ is satisfying $\beta_{k}\left(\bar{\alpha}_{k}^{-1}\left(\delta_{k, b}\right), 0\right)<\bar{\alpha}_{k}^{-1}\left(\delta_{k, x}\right)$, where

$\beta_{k}(\cdot, \cdot)$ is a class $K L$ function.

The continuous bounded control law is constructed as follows [9]

$$
\boldsymbol{u}_{k}(\boldsymbol{x})=-k_{k}\left(\boldsymbol{x}, u_{k}^{\max }\right)\left(L_{\boldsymbol{G}_{k}} V_{k}\right)^{\mathrm{T}}(\boldsymbol{x})
$$

with

$$
\begin{aligned}
& k_{k}\left(\boldsymbol{x}, u_{k}^{\max }\right) \\
& =\left\{\begin{array}{l}
\frac{\alpha_{k}(\boldsymbol{x})+\sqrt{\left(\alpha_{k}^{1}(\boldsymbol{x})\right)^{2}+\left(u_{k}^{\max } \beta_{k}(\boldsymbol{x})\right)^{4}}}{\left(\beta_{k}(\boldsymbol{x})\right)^{2}\left[1+\sqrt{1+\left(u_{k}^{\max } \beta_{k}(\boldsymbol{x})\right)^{2}}\right]}, \\
\left\|\left(L_{\boldsymbol{G}_{k}} V_{k}\right)^{\mathrm{T}}(\boldsymbol{x})\right\| \neq 0 \\
0, \quad\left\|\left(L_{\boldsymbol{G}_{k}} V_{k}\right)^{\mathrm{T}}(\boldsymbol{x})\right\|=0
\end{array}\right.
\end{aligned}
$$

where

$$
\begin{aligned}
& \alpha_{k}(\boldsymbol{x})=L_{f_{k}} V_{k} \\
&+\left(\rho_{k}\left\|2 P_{k} \boldsymbol{e}\right\|+\chi_{k} \theta_{k}^{b}\left\|\left(L_{\boldsymbol{W}_{k}} V_{k}\right)^{\mathrm{T}}\right\|\right) \frac{\left\|2 P_{k} \boldsymbol{e}\right\|}{\left\|2 P_{k} \boldsymbol{e}\right\|+\phi_{k}}, \\
& \beta_{k}(\boldsymbol{x})=\left\|\left(L_{\boldsymbol{G}_{k}} V_{k}\right)^{\mathrm{T}}(\boldsymbol{x})\right\|, \\
& \alpha_{k}^{1}(\boldsymbol{x})=L_{\boldsymbol{f}_{k}} V_{k}+\rho_{k}\left\|2 P_{k} \boldsymbol{e}\right\|+\chi_{k} \theta_{k}^{b}\left\|\left(L_{\boldsymbol{W}_{k}} V_{k}\right)^{\mathrm{T}}\right\|, \\
& L_{\boldsymbol{G}_{k}} V_{k}(\boldsymbol{x})=\left[L_{g_{k}^{1}} V_{k}, \cdots, L_{g_{k}^{m}} V_{k}\right] \text { and } \\
& L_{W_{k}} V_{k}(x)=\left[L_{w_{k}^{1}} V_{k}, \cdots, L_{w_{k}^{m}} V_{k}\right] \text { are row vectors, where }
\end{aligned}
$$

$g_{k}^{i}$ is the ith column of $\boldsymbol{G}_{k}$ and $w_{k}^{i}$ is the ith column of $W_{k} ; \rho_{k}>0, \chi_{k}>1, \phi_{k}>0$ are adjustable parameters.

Remark 1. For convenience, this bounded controller (13)-(14) is redefined as $\boldsymbol{B}_{k}(\boldsymbol{x})$.

Remark 2. Here, the Lyapunov functions used in verifying the switching conditions at any given time, $V_{k}$, are based on $\boldsymbol{x}$. Note that the Lyapunov functions $\bar{V}_{k}$ are in general different from $V_{k}$ used in bounded controllers. For the systems with relative degree $r_{k}=n(k=1, \cdots, p)$, the choice of $\bar{V}_{k}=V_{k}$ is sufficient.

Based on this bounded controller (13)-(14), an estimation of the stability region is computed as

$$
\Omega_{k}\left(u_{k}^{\max }\right)=\left\{\boldsymbol{x} \in R^{n}: V_{k}(\boldsymbol{x}) \leq \delta_{k, x}\right\}
$$

where $\delta_{k, x}>0$ is the largest number for which

$$
\begin{aligned}
& \Phi_{k}\left(u_{k}^{\max }\right) \supseteq \Omega_{k}\left(u_{k}^{\max }\right) \backslash\{0\} \text {, and } \\
& \quad \Phi_{k}\left(u_{k}^{\max }\right)=\left\{\boldsymbol{x} \in X: \alpha_{k}^{1}(\boldsymbol{x}) \leq u_{k}^{\max } \beta_{k}(\boldsymbol{x})\right\} .
\end{aligned}
$$

The robustness property of the bounded controller in (13)-(14) is formalized by the following proposition:

Proposition 2. Consider the system (1) for a fixed value $\sigma(t)=k$. Under the Assumptions 1-4, compute the bounded control law of (13)-(14) using the Lyapunov functions $V_{k}$ and $\rho_{k}>0$, and then give the stability region estimate $\Omega_{k}$. Let $\boldsymbol{u}_{k}(t)=\boldsymbol{u}_{k}(j \Delta)$,

$j \Delta \leq t \leq(j+1) \Delta$, where $\boldsymbol{u}_{k}(j \Delta)=\boldsymbol{B}_{k}(\boldsymbol{x}(j \Delta))$,

$j=0, \cdots, \infty$. Then, given any positive real number $d_{k}$, there exists positive real numbers $\Delta_{k}^{*}, \delta_{k}^{\prime}, \varepsilon_{k}$, such that if $\boldsymbol{x}(0):=\boldsymbol{x}_{0} \in \Omega_{k}$ and $\Delta \in\left(0, \Delta_{k}^{*}\right]$, then

$\boldsymbol{x}(t) \in \Omega_{k} \subseteq X, \forall t \geq 0$, and $\limsup _{t \rightarrow \infty}\|\boldsymbol{x}(t)\| \leq d_{k}$. Furthermore, if $V_{k}\left(x_{0}\right) \leq \delta_{k}^{\prime}$, then $V_{k}(x(\tau)) \leq \delta_{k}^{\prime}$, $\forall \tau \in[0, \Delta)$; if $\delta_{k}^{\prime}<V_{k}\left(\boldsymbol{x}_{0}\right) \leq \delta_{k, x}$, then $\dot{V}_{k}(\boldsymbol{x}(\tau)) \leq-\varepsilon_{k}, \forall \tau \in[0, \Delta)$ and the output of the closedloop system satisfies: $\limsup _{t \rightarrow \infty}|y(t)-v(t)|=0$ (The proof is similar to the proof of Theorem 1 in ref. [9]).

\subsection{Output Feedback Bounded Controller Based on State Estimations and Lyapunov Functions}

In this section, we consider the case when some states of system (1) are not measurable. The bounded controller based on state estimations and Lyapunov functions should be designed and the stable region of initial conditions should be described.

Based on the high-order differential state observer (6)(8), the following presents the output feedback controller used for each mode and characterizes its stability properties:

Proposition 3. Considering the nonlinear system (1), for a fixed mode $\sigma(t)=k(k \in K)$, design the output feedback controller with a high-order differential state observer (6)-(8)

$$
\boldsymbol{u}_{k}=-k_{k}\left(\hat{\boldsymbol{x}}, u_{k}^{\max }\right)\left(L_{\boldsymbol{G}_{k}} V_{k}(\hat{\boldsymbol{x}})\right)^{\mathrm{T}} \triangleq \boldsymbol{B}_{k}(\hat{\boldsymbol{x}})
$$

where $\hat{\boldsymbol{x}}=\chi_{k}^{-1}(\operatorname{sat}(\hat{\boldsymbol{y}})), \hat{\boldsymbol{y}}=\left[\hat{y}_{1}, \hat{y}_{2}, \cdots, \hat{y}_{r_{k}}\right]^{\mathrm{T}}$, 
$\tilde{\boldsymbol{y}}=\left[\tilde{y}_{1}, \tilde{y}_{2}, \cdots, \tilde{y}_{r_{k}}\right]^{\mathrm{T}}$ are vectors with dimension $r_{k}$.

Given $\xi_{\max , k}=\beta_{\xi}\left(\delta_{\xi, k}, 0\right)$, where $\beta_{\xi}$ is a class $K L$ function and $\delta_{\xi, k}$ is the maximum value of

$\left\|\left[h_{k}(\boldsymbol{x}), L_{f_{k}} h_{k}(\boldsymbol{x}), \cdots, L_{f_{k}}^{r_{k}-1} h_{k}(\boldsymbol{x})\right]^{\mathrm{T}}\right\|$ for $V_{k}(x) \leq \delta_{k, b}$, and $L_{k}>0$, such that if $\varepsilon_{k}=1 / L_{k}$, then, given $\Omega_{b, k}$, there exists $\varepsilon_{k}^{*}>0$, such that if $\varepsilon_{k} \in\left(0, \varepsilon_{k}^{*}\right]$,

$\boldsymbol{x}(0) \in \Omega_{k, b}$ and $\|\tilde{\boldsymbol{y}}(0)\| \leq \delta_{\xi, k}$, the origin of the closedloop system is asymptotically (and locally exponentially) stable, i.e., there exists $d_{k}>0$, such that

$\limsup _{t \rightarrow \infty}\|\boldsymbol{x}(t)\| \leq d_{k}$. Furthermore, given $\varepsilon_{k} \in\left(0, \varepsilon_{k}^{*}\right]$ and some real number $e_{m, k}>0$, there exists a real number $T_{k}^{b}>0$ such that $\|\boldsymbol{x}(t)-\hat{\boldsymbol{x}}(t)\| \leq e_{m, k}$, for $\forall t \geq T_{k}^{b}$. And, the output of the closed-loop system satisfies

$\limsup _{t \rightarrow \infty}|y(t)-v(t)|=0$ (This proposition is a special case of Theorem 2 in ref. [9]).

Remark 3. Here, a high order differential state observer is used to provide the estimates of the derivatives of the output $y$ up to order $r_{k}-1$, denoted as $\hat{y}_{1}, \hat{y}_{2}, \cdots, \hat{y}_{r_{k}}$, and thus estimates of the variables $\xi_{1}, \cdots, \xi_{r_{k}}$ (note from Assumption 1 that $\left.\xi_{i}=\frac{\mathrm{d}^{i-1} y}{\mathrm{~d} t^{i-1}}, i=1, \cdots, r_{k}\right)$. This high-order differentiator has only one adjustable parameter. To eliminate the peaking phenomenon associated with the high order differential states observer, a standard saturation function sat (.) is introduced to eliminate wrong estimates of the output derivatives, or alternatively the following formulation can be used [12-14]

$$
\begin{aligned}
\hat{y}_{1} & =\tilde{y}_{1}, \hat{y}_{i}=\left(\tilde{y}_{i}+a_{k, i}\left(y-\tilde{y}_{1}\right)\right) \sigma_{k, i}(t), \\
i & =2, \cdots, r_{k}
\end{aligned}
$$

where $\sigma_{k, i}(t)=\frac{1-\exp \left(-\lambda_{k} t^{2 i}\right)}{1-\exp \left(-\lambda_{k} t^{2 i}\right)}$.

Remark 4. The ith closed-loop subsystem can be cast as a two time-scale system given by

$$
\begin{aligned}
\dot{\boldsymbol{e}}_{0} & =\boldsymbol{A}_{k} \boldsymbol{e}_{0}+\boldsymbol{b} y^{\left(r_{k}\right)} \\
\dot{\boldsymbol{x}} & =\boldsymbol{f}_{k}(\boldsymbol{x})-\boldsymbol{G}_{k}(\boldsymbol{x}) k_{k}\left(\hat{\boldsymbol{x}}, u_{k}^{\max }\right)\left(L_{\boldsymbol{G}_{k}} V_{k}(\hat{\boldsymbol{x}})\right)^{\mathrm{T}} \\
& +\boldsymbol{W}_{k}(\boldsymbol{x}) \boldsymbol{\theta}_{k}
\end{aligned}
$$

where $\boldsymbol{e}_{0}$ is a vector of the auxiliary error variables $\hat{e}_{i}=y^{(i-1)}-\hat{y}_{i}, i=1, \cdots, r_{k}$, and

$$
\boldsymbol{A}_{k}=\left[\begin{array}{ccccc}
-a_{k, 1} & 1 & 0 & \cdots & 0 \\
-a_{k, 2} & 0 & 1 & \cdots & 0 \\
\vdots & \vdots & \vdots & \ddots & \vdots \\
-a_{k, r_{k}-1} & 0 & 0 & \cdots & 1 \\
-a_{k, r_{k}} & 0 & 0 & \cdots & 0
\end{array}\right], \quad \boldsymbol{b}=\left[\begin{array}{c}
0 \\
0 \\
\vdots \\
1
\end{array}\right]
$$

Proposition 4 establishes the existence of a set, $\Omega_{k, s}$, such that once the state estimation error is smaller than a certain value (note that the decay rate can be controlled by adjusting $L_{k}$ ), the presence of the state is within the output feedback stability region, $\Omega_{k, b}$.

Proposition 4. Given any positive real number $\delta_{k, b}$, there exist positive real numbers $e_{m, k}^{*}, \delta_{k, s}$, and a set $\Omega_{k, s}:=\left\{\boldsymbol{x} \in R^{n}: V_{k}(\boldsymbol{x}) \leq \delta_{k, s}\right\}$ such that if $\|\boldsymbol{x}-\hat{\boldsymbol{x}}\| \leq e_{m, k}$, where $e_{m, k} \in\left(0, e_{m, k}^{*}\right]$, then $\hat{\boldsymbol{x}} \in \Omega_{k, s} \Rightarrow \boldsymbol{x} \in \Omega_{k, b}$.

\section{Integrated Predictive Controller Design Based on State Estimation and Lyapunov Functions}

\subsection{Predictive Controller Design Based on State Estimation and Lyapunov Functions for Every Subsystem}

A MPC based on the high-order differential state observer and Lyapunov functions will be designed for system (1) with a fixed $\sigma(t)=k \quad k \in K$ in this section. The control action at time $t$ and state estimation $\hat{x}$ are conventionally obtained by on-line solving a finite horizon optimal control problem described as

$$
\begin{aligned}
& P(\hat{\boldsymbol{x}}, t): \min \left\{J\left(\hat{\boldsymbol{x}}, t, \boldsymbol{u}_{k}(\cdot)\right) \mid \boldsymbol{u}_{k}(\cdot) \in S_{k}\right\} \\
& \sum:\left\{\begin{array}{l}
\dot{\tilde{y}}_{i}=\tilde{y}_{i+1}+a_{k, i}\left(y-\tilde{y}_{1}\right), \quad i=1, \cdots, r_{k}-1 \\
\dot{\tilde{y}}_{r_{k}}=a_{k, r_{k}}\left(y-\tilde{y}_{1}\right)
\end{array}\right. \\
& \left\{\begin{array}{l}
\hat{y}_{1}=\tilde{y}_{1} \\
\hat{\boldsymbol{y}}_{i+1}=\tilde{y}_{i+1}+a_{k, i}\left(y-\tilde{y}_{1}\right), \quad i=1, \cdots, r_{k}-1
\end{array}\right.
\end{aligned}
$$

where

$$
\begin{aligned}
& a_{k, i}=K_{k} C_{r_{k}-1}^{i-1} a_{k}^{i-1}, i=1, \cdots, r_{k} \\
& K_{k}=\frac{\left(r_{k} a_{k}\right)^{r_{k}}}{\left(\left(r_{k}-1\right) a_{k}\right)^{r_{k}-1}}=\frac{r_{k}^{r_{k}} a_{k}}{\left(r_{k}-1\right)^{r_{k}-1}} \\
& \dot{\eta}=\Psi_{k}(\hat{\boldsymbol{y}}, \boldsymbol{\eta}), \hat{\boldsymbol{x}}=\chi_{k}^{-1}(\hat{\boldsymbol{y}}) \\
& \dot{\boldsymbol{x}}=\boldsymbol{f}_{k}(\boldsymbol{x})+\boldsymbol{G}_{k}(\boldsymbol{x}) \boldsymbol{u}_{k}(\hat{\boldsymbol{x}})+\boldsymbol{W}_{k}(\boldsymbol{x}) \boldsymbol{\theta}_{k}, \\
& \quad \boldsymbol{u}_{k}(\hat{\boldsymbol{x}}) \in U_{k} \subseteq R^{m}, \boldsymbol{\theta}_{k} \in \Theta_{k} \subseteq R^{q} \\
& y=h_{k}(\boldsymbol{x}), \\
& \dot{V}_{k}(\hat{\boldsymbol{x}}(\tau)) \leq-\varepsilon_{k}, \tau \in[t, t+\Delta), \\
& \text { if } V_{k}(\hat{\boldsymbol{x}}(t))>\delta_{k}^{\prime} \\
& V_{k}(\hat{\boldsymbol{x}}(\tau)) \leq \delta_{k}^{\prime}, \tau \in[t, t+\Delta), \\
& \text { if } V_{k}(\hat{\boldsymbol{x}}(t)) \leq \delta_{k}^{\prime}
\end{aligned}
$$




$$
\dot{V}_{k}^{c}\left(\hat{\boldsymbol{x}}_{f}(\tau)\right)<0, \quad \tau \in[t, t+\Delta) .
$$

Here $\varepsilon_{k}, \delta_{k}^{\prime}$ are defined in Proposition $1, e_{0}, \hat{\boldsymbol{x}}$ are defined in Proposition 2 and $\boldsymbol{x}_{f}=\left[\boldsymbol{x}^{\mathrm{T}}, \boldsymbol{e}_{0}^{\mathrm{T}}\right]^{\mathrm{T}}$.

$S_{k}=S_{k}\left(t, T_{k}\right)$ is a family of piecewise continuous functions (from the right), with period $\Delta$, mapping $\left[t, t+T_{k}\right]$ into the set of admissible controls $U_{k} \cdot T_{k}$ is the horizon length and $V_{k}$ is the Lyapunov function used to design bounded controller. $V_{k}^{c}$ is the Lyapunov function of the system. A control $\boldsymbol{u}_{k}(\cdot)$ in $S_{k}$ is characterized by the sequence $\left\{\boldsymbol{u}_{k}[j]\right\}, \boldsymbol{u}_{k}[j]=\boldsymbol{u}_{k}(j \Delta)$ and satisfies $\boldsymbol{u}_{k}(t)=\boldsymbol{u}_{k}[j]$ for all $t \in[j \Delta,(j+1) \Delta)$. The performance index is given by

$$
\begin{aligned}
& J\left(\hat{\boldsymbol{x}}, t, \boldsymbol{u}_{k}(\cdot)\right) \\
& =\int_{t}^{t+T_{k}}\left[\hat{\boldsymbol{x}}^{\mathrm{T}}(s) \boldsymbol{Q}_{k} \hat{\boldsymbol{x}}(s)+\boldsymbol{u}_{k}^{\mathrm{T}}(s) \boldsymbol{R}_{k} \boldsymbol{u}_{k}(s)\right] \mathrm{d} s
\end{aligned}
$$

where $Q_{k}$ and $R_{k}$ are positive semi-definite and strictly positive definite symmetric matrices, respectively. The optimal control $\boldsymbol{u}_{k}^{0}(\hat{x}) \in S_{k}$ is then applied to the plant over the interval $[t, t+\Delta)$ and the procedure is repeated indefinitely. This defines an implicit model predictive control law

$$
\boldsymbol{M}_{k}(\hat{\boldsymbol{x}}):=\arg \min \left(J\left(\hat{\boldsymbol{x}}, t, \boldsymbol{u}_{k}(\cdot)\right)\right):=\boldsymbol{u}_{k}^{[1]}
$$

Owing to the existence of parameter uncertainties and constraints, the initial feasibility of the MPC in (32) is not guaranteed. If it is infeasible, the control action is switched to the bounded controller (17). To describe the whole control action, we cast the $k$ th subsystem (1) as a switched system of the form

$$
\begin{aligned}
& \dot{\boldsymbol{x}}=\boldsymbol{f}_{k}(\boldsymbol{x})+\boldsymbol{G}_{k}(\boldsymbol{x}) \boldsymbol{u}_{k}^{[i(t)]}+\boldsymbol{W}_{k}(\boldsymbol{x}) \boldsymbol{\theta}_{k} \\
& y=h_{k}(\boldsymbol{x})
\end{aligned}
$$

where $i(t):[0, \infty) \rightarrow\{1,2\}$ is the switching signal which is assumed to be a piecewise continuous (from the right) function of time. When $i(t)=1$, the control input takes $\boldsymbol{u}_{k}^{[i(t)]}(\hat{\boldsymbol{x}})=\boldsymbol{u}_{k}^{[1]}(\hat{\boldsymbol{x}})$, i.e., the MPC is used; and when $i(t)=2$, it takes $\boldsymbol{u}_{k}^{[i(t)]}(\hat{\boldsymbol{x}})=\boldsymbol{u}_{k}^{[2]}(\hat{\boldsymbol{x}})=\boldsymbol{B}_{k}(\hat{\boldsymbol{x}})$, i.e., the bounded control is used.

Proposition 5. Consider the switched nonlinear system in (33). For a fixed $\sigma(t)=k(k \in K)$, the control action is switched between the model predictive controller (21)(31) and the bounded controller (17). Let $\boldsymbol{x}(0)=\boldsymbol{x}_{0} \in \Omega_{k, b},\|\tilde{\boldsymbol{y}}(0)\| \leq \delta_{k, \xi}$. At the earliest time $t \geq 0$ when the closed-loop system's states under MPC satisfy $V_{k}\left(\hat{\boldsymbol{x}}\left(t^{-}\right)\right)=\delta_{k, b}$, set $T_{s}^{k}=t$; at the earliest time $t \geq 0$ when the states under MPC satisfy $\|\hat{\boldsymbol{x}}(t)\| \leq d_{k}$, set $T_{d}^{k}=t$; at the earliest time $t \geq 0$ when the states under MPC satisfy $\|x(t)-\hat{\boldsymbol{x}}(t)\| \leq e_{k, s}$, set $T_{e}^{k}=t$; at the earliest time $t \geq 0$ when MPC is infeasible, set $T_{\text {inf }}^{k}=t$. Define $T_{\text {switch }}^{k}=\min \left\{T_{s}^{k}, T_{d}^{k}, T_{e}^{k}, T_{\text {inf }}^{k}, T_{\text {design }}^{k}\right\}$, where $T_{\text {design }}^{k}\left(0 \leq T_{\text {design }}^{k}<\infty\right)$ is a designed time arbitrary. Then, the switching rule

$$
i(t)= \begin{cases}1 & 0 \leq t<T_{\text {switch }}^{k} \\ 2 & t \geq T_{\text {switch }}^{k}\end{cases}
$$

guarantees the stability of the closed-loop subsystem (see the proof in Appendix A).

Remark 5. The mixed predictive controller above is designed and implemented using the following steps:

1) For the subsystem (1) with a fixed $\sigma(t)=k(k \in K)$, design the bounded controller (17), and compute the stable region

$$
\Omega_{k, b}=\left\{\boldsymbol{x} \in R^{n}: V_{k}(\boldsymbol{x}) \leq \delta_{k, b}\right\} ;
$$

2) Design the MPC controller given by (21)-(31);

3) Given initial conditions $\boldsymbol{x}_{0} \in \Omega_{k, b}$ and $\|\tilde{\boldsymbol{y}}(0)\| \leq \delta_{k, \xi}$, implement the MPC controller given by (21)-(31) if it is feasible;

4) When the MPC is infeasible $\left(t=T_{i n f}^{k}\right)$, or the state estimation $\hat{\boldsymbol{x}}$ of the closed-loop subsystem reaches the boundary of $\Omega_{k, b}$, i.e., when $\|\hat{\boldsymbol{x}}(t)\|=\delta_{k, b} \quad\left(t=T_{s}^{k}\right)$, or $\hat{\boldsymbol{x}}$ satisfies $\|\hat{\boldsymbol{x}}(t)\| \leq d_{k} \quad\left(t=T_{d}^{k}\right)$, or $\|\boldsymbol{x}(t)-\hat{\boldsymbol{x}}(t)\| \leq e_{k, s} \quad\left(t=T_{e}^{k}\right)$, the controller switches to the bounded controller given by (17) until the MPC is feasible again or until the designed switched time $T_{\text {design }}^{k}$;

5) When another subsystem is switched in, go to step 1).

Remark 6. The purpose of switching to the bounded robust controller after the time $T_{d e s i g n}^{k}$ is to ensure convergence and avoid possible cases where the closed-loop states, under MPC, could wander inside $\Omega_{k, b}$ without actual convergence.

\subsection{Integrated Design of the Controller and the Switched Law for the Whole Switched System}

Consider the constrained switched nonlinear system (1) with parameter uncertainties and definite switched time sequences $T_{k, \text { in }}=\left\{t_{k_{1}^{\text {in }}}, t_{k_{2}^{\text {in }}}, \cdots\right\}$ and $T_{k, \text { out }}=\left\{t_{k_{1}^{\text {out }}}, t_{k_{2}^{\text {out }}}, \cdots\right\}$. Theorem 1 gives the switched law that ensures the stability of whole closed-loop system.

Theorem 1. Consider the switched nonlinear system (1) for which there exist control Lyapunov functions $V_{k}$, $k=1, \cdots, p$. Given any initial conditions $\boldsymbol{x}(0) \in \Omega_{k, b}$ and $\|\tilde{\boldsymbol{y}}(0)\| \leq \delta_{k, \xi}$, where $\delta_{k, \xi}$ was defined in Proposition 1 , and chosen positive real numbers $\rho_{k}, k=1, \cdots, p$, compute the stable region estimation $\Omega_{k}$. Choose $\delta_{k, b}>0$ to compute $\Omega_{k, b}$. Choose $M_{k}$ such that 
$\left\|z_{1}-z_{2}\right\| \leq e_{m, k} \Rightarrow\left|V_{k}^{c}\left(z_{1}\right)-V_{k}^{c}\left(z_{2}\right)\right| \leq M_{k}$. Let

$T_{\text {design }}\left(0<T_{\text {design }}<\infty\right)$ be the design time and $t$ satisfy

$t_{k_{r}^{\text {in }}} \leq t \leq t_{k_{r}^{\text {out }}}$. Assume $t_{m_{j}^{\text {in }}}=t_{k_{r}^{\text {out }}}$ for some $m, k$. The

mixed controller switched between the bounded controller (17) and the MPC (21)-(31) is designed with the switched law (34), such that if $t_{k_{r}^{\text {out }}}=t_{m_{j}^{\text {in }}}<\infty$

$$
\begin{aligned}
& V_{m}\left(x\left(t_{m_{j}^{i_{j}}}\right)\right) \\
& \leq \begin{cases}V_{m}\left(\boldsymbol{x}\left(t_{m_{j-1}^{i n}}\right)\right)-\varepsilon^{*}, & \text { if } j>1, V_{m}\left(\boldsymbol{x}\left(t_{m_{j-1}^{\text {in }}}\right)\right)>\delta_{m}^{\prime} \\
\delta_{m}^{\prime}, & \text { if } j>1, V_{m}\left(\boldsymbol{x}\left(t_{m_{j-1}^{\text {in }}}\right)\right) \leq \delta_{m}^{\prime} \\
\delta_{m, b}, & \text { if } j=1\end{cases} \\
& \tilde{\boldsymbol{y}}\left(t_{m_{j}^{\text {in }}}\right)= \begin{cases}\tilde{\boldsymbol{y}}\left(t_{m_{j}^{\text {in }}}\right), & \text { if }\left\|\tilde{\boldsymbol{y}}\left(t_{m_{j}^{\text {in }}}\right)\right\| \leq \delta_{j, \xi} \\
\tilde{\boldsymbol{y}}\left(t_{m_{j}^{\text {in }}}\right) \frac{\delta_{j, \xi}}{\left\|\tilde{\boldsymbol{y}}\left(t_{m_{j}^{i n}}\right)\right\|}, & \text { if }\left\|\tilde{\boldsymbol{y}}\left(t_{m_{j}^{\text {in }}}\right)\right\|>\delta_{j, \xi}\end{cases} \\
& V_{m}^{c}\left(\boldsymbol{x}\left(t_{m_{j}^{i n}}\right)\right)+2 M_{m}<V_{m}^{c}\left(\boldsymbol{x}\left(t_{m_{j-1}^{i n}}\right)\right)
\end{aligned}
$$

then the whole closed-loop system is stable (See the proof in Appendix B).

Remark 7. The controller presented in Theorem 1 can be implemented using the following steps:

1) Given the system model (1) with constraints on the inputs, and a control Lyapunov function $V_{k}$ to design the bounded controller (17) with suitable parameters and compute the stability regions (15) and (16). Here the stability regions are only signs, for the states cannot be measured, and in the controller design only the stable region estimation $\Omega_{k}(\hat{\boldsymbol{x}})$ is used. And choose Lyapunov function $V_{k}^{c}$ for the system (19);

2) Determine suitable parameters to design the MPC in (21)-(31). Give the size of the ball to which the state is required to converge, $d^{\max }$, and compute

$\Delta_{k}^{*}, k=1, \cdots, p$ such that for every subsystem it has $\limsup _{t \rightarrow \infty}\|\boldsymbol{x}(t)\| \leq d^{\max }$. Compute $\Delta^{*}=\min _{k=1}^{p}\left\{\Delta_{k}^{*}\right\}$, and choose $\Delta_{k_{r}} \in\left(0, \Delta^{*}\right]$, for a real positive number $l_{k_{r}}>0$ such that $t_{k_{r}^{\text {out }}}-t_{k_{r}^{\text {in }}}=l_{k_{r}} \Delta_{k_{r}}$;

3) For time $t_{k_{r}^{i n}}$ (the $r$ th time of switching into the $k$ th subsystem), consider whether the state estimation belongs to the stable region $\Omega_{b, k}$;

4) Pick $t_{m_{j}^{\text {in }}}=t_{k_{r}^{\text {out }}}$ in the switched sequence;

5) At the time of switching in the $m$ th subsystem, consider the constraints in Theorem 1 , and choose $\tilde{\boldsymbol{y}}$ and $M_{m}$ satisfying (36) and (37), respectively;
6) When the $m$ th subsystem is switched in, the constraint $V_{m}\left(\hat{\boldsymbol{x}}\left(t_{m_{j}^{\text {in }}}\right)\right) \leq V_{m}\left(\hat{\boldsymbol{x}}\left(t_{m_{j-1}^{\text {in }}}\right)\right)-\varepsilon^{*}$ is required to be satisfied. If $j=1, V_{m}\left(\hat{\boldsymbol{x}}\left(t_{m_{j-1}^{i n}}\right)\right)=\delta_{m, b}$; if the state is in the neighborhood of origin, then $V_{m}\left(\hat{\boldsymbol{x}}\left(t_{m_{j}^{\text {in }}}\right)\right) \leq \delta_{m}^{\prime}$ and $\hat{\boldsymbol{x}} \in \Omega_{m, s}$. If constraint (37) is used to ensure $\|\boldsymbol{x}(t)-\hat{\boldsymbol{x}}(t)\| \leq e_{m, s}$, then $\boldsymbol{x} \in \Omega_{m, b}$ and the closed-loop system is stable according to Proposition 4 .

Remark 8. [10]. The time interval between two consecutive switches should be long enough to ensure that the estimation error decreased to a sufficiently small value such that the closed-loop system is stable. Furthermore, the decision to switch is not based on $\boldsymbol{x} \rightarrow \Omega_{k, b}$, but rather based on $\hat{\boldsymbol{x}} \rightarrow \Omega_{k, s}$ (under state feedback it was based on $\boldsymbol{x} \rightarrow \Omega_{k, b}$ ). If $\hat{\boldsymbol{x}} \rightarrow \Omega_{k, s}$ at some early time, a switch is not executed before $\|\boldsymbol{x}-\hat{\boldsymbol{x}}\| \leq e_{m, s}$.

\section{Simulation}

Consider a continuously stirred tank reactor where three parallel, irreversible, first-order exothermic reactions of the form $A \stackrel{k_{1}}{\longrightarrow} D, A \stackrel{k_{2}}{\longrightarrow} U, A \stackrel{k_{3}}{\longrightarrow} R$ take place, where $A$ is the reactant species, and $D$ is the desired product species, $U, R$ denote the by-product species. Under standard modeling assumptions, the mathematical model for the process takes the form [8]

$$
\begin{aligned}
& \dot{C}_{A}=\frac{F_{\sigma}}{V}\left(C_{A 0}-C_{A}\right)-k_{0} \mathrm{e}^{(-E / R T)} C_{A} \\
& \dot{T}=\frac{F_{\sigma}}{V}\left(T_{A 0}-T\right)+\frac{(-\Delta H)}{\rho C_{p}} k_{0} \mathrm{e}^{(-E / R T)} C_{A}+\frac{Q_{\sigma}}{\rho C_{p} V} \\
& \dot{C}_{D}=-\frac{F_{\sigma}}{V} C_{D}+k_{0} \mathrm{e}^{(-E / R T)} C_{A}
\end{aligned}
$$

where $C_{A}$ and $C_{D}$ denote the concentration of species $A$ and $D$, respectively. $T$ denotes the temperature of reactor. $Q_{\sigma}$ is the rate of heat input to the reactor. $V$ the volume of the reactor. $k_{0}, E, \Delta H$ denote the pre-exponential constants, the activation energies. And the enthalpies of the three reactions, respectively, $c_{p}$ and $\rho$ are the heat capacity and density of the fluid in the reactor. $\sigma(t) \in\{1,2\}$ is the switched variable. The values of these parameters can be found in Table 1.

The control objectives are to: (1) stabilize the reactor temperature at the open-loop unstable steady state $T_{s}=$ $388.58 \mathrm{~K}$ of mode 1 , and (2) maintain the temperature at this steady-state when the reactor switches to mode 2 . The control objective is to be accomplished in the presence of: (1) exogenous time-varying disturbances in the feed stream temperature, (2) parametric uncertainty in 
Table 1. Process parameters and steady-state values.

\begin{tabular}{cccccc}
\hline Process parameters & values & Process parameters & values & Process parameters & values \\
\hline$V$ & $1000.0 \mathrm{~L}$ & $\Delta H$ & $-4.78 \times 10^{4} \mathrm{~J} / \mathrm{mol}$ & $T_{s \sigma}(\sigma=1,2)$ & $388.58 \mathrm{~K}$ \\
$R$ & $8.314 \mathrm{~J} / \mathrm{mol} \cdot \mathrm{K}$ & $k_{0}$ & $1.2 \times 10^{4} \mathrm{~min}^{-1}$ & $C_{A s 1}$ & $3.58 \mathrm{~mol} / \mathrm{L}$ \\
$C_{A 0 s}$ & $4.0 \mathrm{~mol} / \mathrm{l}$ & $E$ & $8.314 \times 10^{4} \mathrm{~J} / \mathrm{mol}$ & $C_{A s 2}$ & $4.55 \mathrm{~mol} / \mathrm{L}$ \\
$T_{A 0 S}$ & $300.0 \mathrm{~K}$ & $\rho$ & $1000.0 \mathrm{~g} / \mathrm{L}$ & $C_{D s 1}$ & $0.42 \mathrm{~mol} / \mathrm{L}$ \\
$F_{1}$ & $3.34 \times 10^{-3} \mathrm{~L} / \mathrm{sec}$ & $C_{p}$ & $0.231 \mathrm{~J} / \mathrm{g} \cdot \mathrm{K}$ & $C_{D s 2}$ & $1.0 \mathrm{~mol} / \mathrm{L}$ \\
$F_{2}$ & $1.67 \times 10^{-3} \mathrm{~L} / \mathrm{sec}$ & & & \\
\hline
\end{tabular}

the enthalpy of the three reactions, and (3) hard constraints on the manipulated inputs.

Note that, with this requirement, both closed-loop modes share the same steady-state temperature but have different steady state reactant concentrations. The control objective is to be accomplished by manipulating Q provided by the jacket, subject to the input constraint $|Q| \leq 25 \mathrm{KJ} / \mathrm{sec}$.

Defining $\theta_{1}=\Delta H-\Delta H_{0}, \quad \theta_{2}=T_{A 0}-T_{A 0 s}, y=x_{1}$, the process model of Equation (34) can be cast in the form of Equation (1)

$$
\begin{aligned}
x(t) & =f_{\sigma(t)}(x(t))+G_{\sigma(t)}(x(t)) u_{\sigma(t)}(t) \\
& +W_{\sigma(t)}(x(t)) \theta_{\sigma(t)}(t) \\
y(t) & =h_{\sigma(t)}(x)
\end{aligned}
$$

where

$$
\begin{aligned}
& f_{\sigma}(x)=\left[\begin{array}{c}
-\frac{F_{\sigma}}{V} x_{1}+k_{10} \mathrm{e}^{\left(-E / R x_{3}\right)} x_{2} \\
\frac{F_{\sigma}}{V}\left(C_{A 0}-x_{2}\right)-k_{0} \mathrm{e}^{\left(-E / R x_{3}\right)} x_{2} \\
\frac{F_{\sigma}}{V}\left(T_{A}-x_{3}\right)+\frac{(-\Delta H)}{\rho c_{p}} k_{0} \mathrm{e}^{\left(-E / R x_{3}\right)} x_{2}
\end{array}\right],(\sigma=1,2), \\
& g_{1}(x)=g_{2}(x)=\left[\begin{array}{c}
0 \\
0 \\
\frac{1}{\rho c_{p} V}
\end{array}\right], h_{1}(x)=h_{2}(x)=x_{1}, \\
& \omega_{1}(x)=\left[\begin{array}{c}
0 \\
0 \\
k_{0} \mathrm{e}^{\left(-E / R x_{3}\right)} x_{2}
\end{array}\right], \quad w_{2}(x)=\left[\begin{array}{c}
0 \\
0 \\
F_{1}
\end{array}\right] .
\end{aligned}
$$

The boundary of parameters is $\theta_{b 1}=0.5\left|\left(-\Delta H_{k 0}\right)\right|$, $\theta_{b 2}=0.03 T_{A 0 s}$. For this system, perform the following coordinate transformation

$$
\begin{aligned}
& {\left[\begin{array}{c}
e_{\sigma 1} \\
e_{\sigma 2} \\
e_{\sigma 3}
\end{array}\right]=\left[\begin{array}{c}
\xi_{\sigma 1}-v \\
\xi_{\sigma 2}-v \\
\xi_{\sigma 3}-v^{(1)}
\end{array}\right]=\left[\begin{array}{c}
x_{3}-v \\
x_{1}-v \\
-\frac{F_{\sigma}}{V} x_{1}+k_{0} \mathrm{e}^{\left(-E / R x_{3}\right)} x_{2}
\end{array}\right],} \\
& (\sigma=1,2)
\end{aligned}
$$

Two quadratic, positive-definite functions of the form, $\bar{V}_{1}=\frac{1}{2 T_{s 1}^{2}} e_{1}^{2}, \bar{V}_{2}=\frac{1}{2 T_{s 2}^{2}} e_{2}^{2}$, are then used to synthesize two bounded nonlinear controllers (one for each mode) of the form

$$
\begin{aligned}
& u_{k}(x) \\
& =-\frac{L_{\bar{f}_{i}} \bar{V}_{i}(x)+\rho V_{i}+\sqrt{\left(L_{\bar{f}_{i}} \bar{V}_{i}(x)+\rho V_{i}\right)^{2}+\left(u_{i}^{\max } L_{\bar{g}_{i}} \bar{V}_{i}\right)^{4}}}{\left(L_{\bar{g}_{i}} \bar{V}\right)^{2}\left[1+\sqrt{1+\left(u_{i}^{\max } L_{\bar{g}} \bar{V}\right)^{2}}\right]} \\
& \cdot L_{\bar{g}_{i}} \bar{V}, \\
& i=1,2
\end{aligned}
$$

Note that these positive-define function is given for system (39). To estimate the stability regions, the Lyapunov functions

$$
\begin{aligned}
V_{1}= & \frac{1}{2} c_{1}\left(\left(T-T_{s 1}\right) / T_{s 1}\right)^{2}+\frac{1}{2} c_{2}\left(\left(C_{A}-C_{A s 1}\right) / C_{A s 1}\right)^{2} \\
& +\frac{1}{2} c_{3}\left(\left(C_{D}-C_{D s 1}\right) / C_{D s 1}\right)^{2}
\end{aligned}
$$

where $c_{1}=38.8, c_{2}=1.0, c_{3}=0.6$; and

$$
\begin{aligned}
V_{2}= & \frac{1}{2} c_{4}\left(\left(T-T_{s 2}\right) / T_{s 2}\right)^{2}+\frac{1}{2} c_{5}\left(\left(C_{A}-C_{A s 2}\right) / C_{A s 2}\right)^{2} \\
& +\frac{1}{2} c_{6}\left(\left(C_{D}-C_{D s 2}\right) / C_{D s 2}\right)^{2}
\end{aligned}
$$

where $c_{3}=19.4, c_{4}=1.0, c_{3}=0.3$, are used.

Given initial state $x_{0}=[0.5 \mathrm{~mol} / \mathrm{L}, 2.20 \mathrm{~mol} / \mathrm{L}, 300 \mathrm{~K}]^{\mathrm{T}}$, and switched time $T_{\text {design }}=30 \mathrm{sec}$, the parameters of the high-order differential state observer take as 
$a_{1}=0.9, a_{2}=1.5$. Using the hybrid MPC method proposed in this paper, we obtained the following simulation results:

In the simulation, starting from mode 1, the predictive controller was first implemented before switching to the bounded controller at $t=21.3 \mathrm{sec}$. At the designed switched time $T_{\text {design }}=30 \mathrm{sec}$ the system turned to mode 2 . In mode 2, the predictive controller was first implemented before switching to the bounded controller at $t=64.56 \mathrm{sec}$, and at $t=73.87 \mathrm{sec}$ it was again switched to predictive controller.

Figures 1 to $\mathbf{4}$ demonstrate the validity of the controller proposed in this paper.

\section{Conclusion}

In this paper, a hybrid predictive control method is pro-

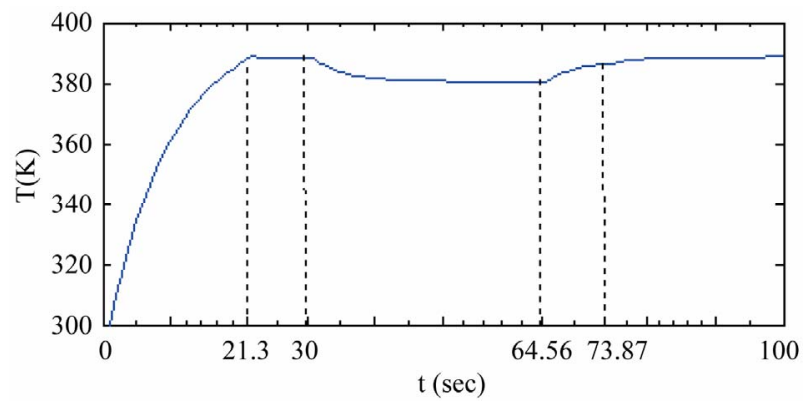

Figure 1. Closed-loop state (the reactor temperature $T$ ) profile.

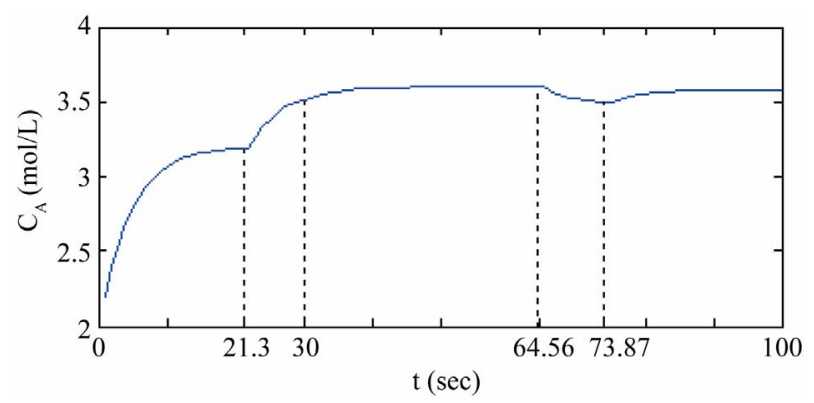

Figure 2. Closed-loop state (the reactor concentration $C_{A}$ ) profile.

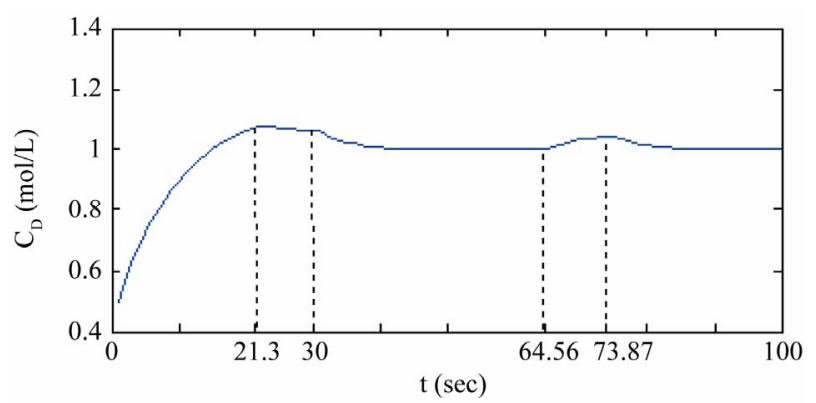

Figure 3. Closed-loop state (the reactor concentration $C_{D}$ ) profile.

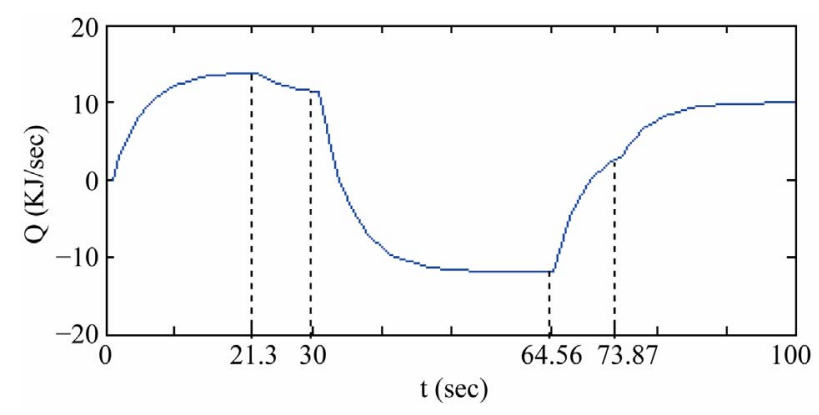

Figure 4. The input $Q$ profile.

posed for a class of uncertain switched nonlinear systems with input constraints and unavailable state measurements. The main objectives were to design a hybrid controller which switches between a bounded controller and a predictive controller based on Lyapunov functions and a high-order differential state observer with a suitable switched law to stabilize the closed-loop subsystem, and to provide an explicitly characterized set of initial conditions. For the whole switched system, a suitable switched law based on the state estimation was derived to ensure the whole closed-loop system's stability. The simulation results for a continuously stirred tank reactor showed the validity of the controller proposed in this paper.

\section{Acknowledgements}

This work was supported by the National Natural Science Foundation of Peoples Republic of China under Grants 61374004, 61004013, 61104007 and 60804033, the National Specialized Research Fund for the Doctoral Program of Higher Education under Grant 20113705120003, Higher Educational Science and Technology Program Foundation of Shandong Province under Grant J10LG28, J11LG08 and the Doctoral Starting Research Fund from the Qufu Normal University.

\section{REFERENCES}

[1] J. Hespanha and A. S. Morse, "Switching between Stabilizing Controllers,” Automatica, Vol. 38, No. 11, 2002, pp. 1905-1917. doi:10.1016/S0005-1098(02)00139-5

[2] S. L. D. Kothare and M. Morari, "Contractive Model Predictive Control for Constrained Nonlinear Systems," IEEE Transactions on Automatic Control, Vol. 45, No. 6, 2000, pp. 1053-1071. doi:10.1109/9.863592

[3] D. Q. Mayne, J. B. Rawlings and P. O. M. Rao, “Constrained Model Predictive Control: Stability and Optimality,” Automatica, Vol. 36, No. 6, 2000, pp. 789-814. doi:10.1016/S0005-1098(99)00214-9

[4] M. S. Branicky, "Multiple Lyapunov Functions and Other Analysis Tools for Switched and Hybrid Systems," IEEE Transactions on Automatic Control, Vol. 43, No. 4, 1998, pp. 475-482. doi:10.1109/9.664150

[5] P. Mhaskar, N. H. El-Farra and P. D. Christofides, "Ro- 
bust Hybrid Predictive Control of Nonlinear Systems,” Automatica, Vol. 41, No. 2, 2005, pp. 209-217. doi:10.1016/j.automatica.2004.08.020

[6] P. Mhaskar, N. H. El-Farra and P. D. Christofides, "Stabilization of Nonlinear Systems with State and Control Constraints Using Lyapunov-Based Predictive Control," Systems and Control Letters, Vol. 55, No. 8, 2006, pp. 650659. doi:10.1016/j.sysconle.2005.09.014

[7] P. Mhaskar, N. H. El-Farra and P. D. Christofides, "Predictive Control of Switched Nonlinear Systems with Scheduled Mode Transitions,” IEEE Transactions on Automatic Control, Vol. 50, No. 11, 2005, pp. 1670-1680. doi:10.1109/TAC.2005.858692

[8] S. Baili and L. Shaoyuan, "Constrained Predictive Control for Nonlinear Switched Systems with Uncertainty," Acta Automatica Sinica, Vol. 34, No. 9, 2008, pp. 11411147

[9] N. H. E1-Farra and P. D. Christofides, "Bounded Robust Control of Constrained Multivariable Nonlinear Processes," Chemical Engineering Science, Vol. 58, No. 13, 2003, pp. 3025-3047. doi:10.1016/S0009-2509(03)00126-X
[10] N. H. E1-Farra, P. Mhaskar and P. D. Christofides, "Output Feedback Control of Switched Nonlinear Systems Using Multiple Lyapunov Functions," Systems \& Control Letters, Vol. 54, No. 12, 2005, pp. 1163-1182. doi:10.1016/j.sysconle.2005.04.005

[11] B. L. Su, S. Y. Li and Q. M. Zhu, “The Design of Predictive Control with Characterized Set of Initial Condition for Constrained Switched Nonlinear System," Science in China Series E-Technological Sciences, Vol. 52, No. 2, 2009, pp. 456-466. doi:10.1007/s11431-008-0249-8

[12] G. Y. Qi, Z. Chen and Z. Yuan, "Adaptive High Order Differential Feedback Control for Affine Nonlinear System," Chaos, Solitons \& Fractals, Vol. 37, 2008, pp. 308-315. doi:10.1016/j.chaos.2006.09.027

[13] G. Y. Qi, Z. Chen and Z. Yuan, "Model Free Control of Affine Chaotic System,” Physics Letters A, Vol. 344, No. 2-4, 2005, pp 189-202. doi:10.1016/j.physleta.2005.06.073

[14] G. Y. Qi, M. A. van Wyk and B. J. van Wyk, "Model-Free Differential States Observer for Nonlinear Affine System," The 7th IFAC Symposium on Nonlinear Control Systems, 21-24 August 2007, Pretoria, pp. 984-989 


\section{Appendix A}

\section{Proof of Proposition 4}

The proof uses the result of Proposition 3, if the bounded controller (17) is switched to, the state estimation of the closed-loop system resides in $\Omega_{k, b}$, i.e., there exist $\delta_{k, s}>0, e_{m, s}>0$, such that $\hat{\boldsymbol{x}}\left(T_{\text {switch }}^{k}\right) \in \Omega_{k, s}$. Here, the high-order differential state observer is used to estimate states of the controlled system; it is able to converge to the state evolution of the controlled system. So we can have $\|\boldsymbol{x}(t)-\hat{\boldsymbol{x}}(t)\| \leq e_{m, b}$, then $\forall t \geq T_{\text {switch }}^{k}$, using the result of Proposition 4 , we have $\boldsymbol{x}(t) \in \Omega_{k, b}, \forall t \geq T_{\text {switch }}^{k}$ and $\|\boldsymbol{x}\| \leq d_{k}$.

Therefore, we need only show that under the MPC (21)-(31), the closed-loop system is stable until the bounded controller is switched to. In order to do this, we consider five possible values of $T_{\text {switch }}^{k}$ to show that $\hat{\boldsymbol{x}}\left(T_{s w i t c h}^{k}\right) \in \Omega_{k, s}$. Owing to the constraints (28)-(29), if $\hat{\boldsymbol{x}}\left(T_{s w i t c h}^{k}\right) \in \Omega_{k, s}$, we can have $\hat{\boldsymbol{x}}(t) \in \Omega_{k, s}$. Since $\|\boldsymbol{x}(t)-\hat{\boldsymbol{x}}(t)\| \leq e_{m, b}$, we can have $\boldsymbol{x}(t) \in \Omega_{k, b}$, so the closed-loop subsystem is stable.

Now, we show $\hat{\boldsymbol{x}}\left(T_{\text {switch }}^{k}\right) \in \Omega_{k, s}$ for all the possible values of $T_{\text {switch }}^{k}$.

Case 1. If $T_{\text {switch }}^{k}=T_{s}^{k} \quad\left(T_{s}^{k}\right.$ is the earliest time when states under MPC satisfy $\left.V_{k}\left(\hat{\boldsymbol{x}}\left(t^{-}\right)\right)=\delta_{k, b}\right)$, then we can have $\hat{\boldsymbol{x}}\left(\left(T_{s}^{k}\right)^{-}\right) \in \Omega_{k, s}$. By continuity of the solution of system (1), we have $\hat{\boldsymbol{x}}\left(T_{s}^{k}\right) \in \Omega_{k, s}$, i.e., $\hat{\boldsymbol{x}}\left(T_{\text {switch }}^{k}\right) \in \Omega_{k, s}$.

Case 2. If $T_{\text {switch }}^{k}=T_{d}^{k} \quad\left(T_{d}^{k}\right.$ is the earliest time when states satisfy $\|\hat{\boldsymbol{x}}(t)\| \leq d_{k}$ ), as long as $d_{k}$ is small enough, we can have $\hat{\boldsymbol{x}}\left(T_{d}^{k}\right) \in \Omega_{k, s}$, i.e., $\hat{\boldsymbol{x}}\left(T_{\text {switch }}^{k}\right) \in \Omega_{k, s}$.

Case 3. If $T_{\text {switch }}^{k}=T_{\text {design }}^{k}$, then from the definition of $T_{\text {switch }}^{k}$, we can have $T_{\text {design }}^{k} \leq T_{s}^{k}$. We proceed by contradiction to prove $\hat{\boldsymbol{x}}\left(T_{\text {design }}^{k}\right) \in \Omega_{k, s}$. Assume

$\hat{\boldsymbol{x}}\left(T_{\text {design }}^{k}\right) \notin \Omega_{k, s}$, then $V_{k}\left(\hat{\boldsymbol{x}}\left(T_{\text {design }}^{k}\right)\right)>\delta_{k, s}$. Owing to continuity of the solution and $V_{k}(\cdot)$, also since the fact $\hat{x}(0) \in \Omega_{k, s}=\left\{x \in R^{n}: V_{k}(x) \leq \delta_{k, s}\right\}$, there exists a time
$T_{s}^{k^{\prime}}\left(0 \leq T_{s}^{k^{\prime}} \leq T_{\text {design }}^{k}\right)$ for which $V_{k}\left(\hat{\boldsymbol{x}}\left(T_{s}^{k^{\prime}}\right)\right)=\delta_{k, s}$. Since $T_{s}^{k}$ is the earliest time for which $V_{k}\left(\hat{\boldsymbol{x}}\left(T_{s}^{k-}\right)\right)=\delta_{k, s}$, it must be true that $T_{s}^{k} \leq T_{s}^{k^{\prime}} \leq T_{\text {design }}^{k}$, which leads to a contradiction with $T_{\text {design }}^{k} \leq T_{s}^{k}$. Therefore we can have $\hat{\boldsymbol{x}}\left(T_{\text {switch }}^{k}\right) \in \Omega_{k, s}$.

Case 4 and Case 5. If $T_{\text {switch }}^{k}=T_{e}^{k}$ or $T_{\text {switch }}^{k}=T_{\text {inf }}^{k}$, we can prove $\hat{\boldsymbol{x}}\left(T_{\text {switch }}^{k}\right) \in \Omega_{k, s}$ similar to Case 3 only need to replace $T_{\text {design }}^{k}$ by $T_{e}^{k}$, and $T_{i n f}^{k}$, respectively.

By Proposition 4, we have $\boldsymbol{x}(t) \in \Omega_{k, s}, \forall t \geq T_{\text {switch }}^{k}$, and $\|\boldsymbol{x}\| \leq d_{k}$. This completes the proof of Proposition 5.

\section{Appendix B}

Proof of Theorem 1. (Similar to the proof of Theorem in ref. [7])

Based on Propositions 3-5, we need only to prove that, with the switched law (35)-(37), the whole closed-loop system is still stable.

Let $t$ satisfy $t_{k_{r}^{\text {in }}} \leq t<t_{k_{r}^{\text {out }}}$ and $t_{m_{j}^{\text {in }}}=t_{k_{r}^{\text {out }}}<\infty$. For the active mode $k$, constraint (35) ensures the initial conditions switched on mode $k$, using the result of Proposition 5, we can have the mode $k$ is stable. So we need only to prove the stability at the switched time.

If $V_{k}(\hat{\boldsymbol{x}})>\delta_{k}^{\prime}$, using the constraint (28), we can have $V_{k}\left(\hat{\boldsymbol{x}}\left(t_{k_{r}^{\text {out }}}\right)\right)<V_{k}\left(\hat{\boldsymbol{x}}\left(t_{k_{r}^{\text {in }}}\right)\right)$. While the constraint (35) ensures that $V_{k}\left(\hat{\boldsymbol{x}}\left(t_{k_{r+1}^{i n}}\right)\right)<V_{k}\left(\hat{\boldsymbol{x}}\left(t_{k_{r}^{i n}}\right)\right)$ if this mode is switched out and then switched back in. So we can have $V_{k}\left(\hat{\boldsymbol{x}}\left(t_{k_{l}^{i n}}\right)\right)<V_{k}\left(\hat{\boldsymbol{x}}\left(t_{k_{l-1}^{i n}}\right)\right)<\cdots<\delta_{k, b}$. Owing to the feasibility of constraints (28)-(29), then the value of $V_{k}(\hat{\boldsymbol{x}})$ continuously decreases. If this mode is not switched in, there exists at lease some $j \in 1, \cdots, p$ such that mode $j$ is active and Lyapunov function $V_{j}$ continues to decrease until $V_{j} \leq \delta_{j}^{\prime}$. Similar to discussion before, the constraint (35) ensures that $V_{j}$ continues to be less than $\delta_{j}^{\prime}$. Hence, $\limsup _{t \rightarrow \infty}\|\hat{\boldsymbol{x}}(t)\| \leq d^{\max }$. The switched condition (37) ensures the boundedness of $\|\boldsymbol{x}(t)-\hat{\boldsymbol{x}}(t)\|$ at switched transition time, so $\limsup _{t \rightarrow \infty}\|\boldsymbol{x}(t)\| \leq d^{\max }$. This completes the proof of Theorem 1. 